Guzewich Scott, D. (Orcid ID: 0000-0003-1149-7385)

Martinez German (Orcid ID: 0000-0001-5885-236X)

Newman Claire (Orcid ID: 0000-0001-9990-8817)

Baker Mariah, MacQueen (Orcid ID: 0000-0002-2361-1045)

Hassler Donald M. (Orcid ID: 0000-0001-8830-1200)

Kahanp\&\#x00E4;\&\#x00E4; Henrik, T. (Orcid ID: 0000-0001-9108-186X)

Smith Michael, D. (Orcid ID: 0000-0002-7601-1158)

Sullivan Robert (Orcid ID: 0000-0003-4191-598X)

de la Torre Juarez Manuel (Orcid ID: 0000-0003-1393-5297)

Vasavada Ashwin, R. (Orcid ID: 0000-0003-2665-286X)

Vi\&\#x00FA;dez-Moreiras Daniel (Orcid ID: 0000-0001-8442-3788)

Zeitlin Cary (Orcid ID: 0000-0002-1737-141X)

Zorzano Maria-Paz (Orcid ID: 0000-0002-4492-9650)

\title{
Mars Science Laboratory Observations of the 2018/Mars Year 34 Global Dust Storm
}

\author{
Guzewich, Scott D. (301-286-1542) \\ NASA Goddard Spaceflight Center \\ 8800 Greenbelt Road, Code 693 \\ Greenbelt, MD 20771
}

M. Lemmon

Space Science Institute

College Station, TX 77840

C.L. Smith

York University

Department of Earth and Space Science and Engineering

Toronto, ON, Canada M3J 1P3

G. Martínez

University of Michigan

College of Engineering

2455 Hayward Street

Ann Arbor, MI 48109

Á. de Vicente-Retortillo

University of Michigan

College of Engineering

2455 Hayward Street

Ann Arbor, MI 48109

This is the author manuscript accepted for publication and has undergone full peer review but has not been through the copyediting, typesetting, pagination and proofreading process, which may lead to differences between this version and the Version of Record. Please cite this article as doi: 10.1029/2018GL080839

This article is protected by copyright. All rights reserved. 
C.E. Newman

Aeolis Research

Pasadena, CA 91107

M. Baker

Johns Hopkins University

Department of Earth and Planetary Science

Baltimore, MD 21218

C. Campbell

York University

Department of Earth and Space Science and Engineering

Toronto, ON, Canada M3J 1P3

B. Cooper

York University

Department of Earth and Space Science and Engineering

Toronto, ON, Canada M3J 1P3

J. Gómez-Elvira

Centro de Astrobiología (INTA-CSIC)

Torrejón de Ardoz

28850 Madrid, Spain

A.-M. Harri

Finnish Meteorological Institute

Helsinki, Finland

D. Hassler

Southwest Research Institute

Boulder, CO 80302

\section{F. J. Martin-Torres}

Atmospheric Science Group, Division of Space Technology, Department of Computer Science, Electrical and Space Engineering

Luleå University of Technology

Luleå, Sweden

Instituto Andaluz de Ciencias de la Tierra (CSIC-UGR)

Armilla, Granada, Spain

This article is protected by copyright. All rights reserved. 
T. McConnochie

University of Maryland

Department of Astronomy

College Park, MD 20742

J.E. Moores

York University

Department of Earth and Space Science and Engineering

Toronto, ON, Canada M3J 1P3

H. Kahanpää

Finnish Meteorological Institute

Helsinki, Finland

Aalto University

School of Electrical Engineering

Espoo, Finland

A. Khayat

CRESST II and University of Maryland

Department of Astronomy

College Park, MD 20742

NASA Goddard Spaceflight Center

8800 Greenbelt Road, Code 693

Greenbelt, MD 20771

M.I. Richardson

Aeolis Research

Pasadena, CA 91107

M.D. Smith

NASA Goddard Spaceflight Center

8800 Greenbelt Road, Code 693

Greenbelt, MD 20771

R. Sullivan

Cornell Center for Astrophysics and Planetary Science

Cornell University

This article is protected by copyright. All rights reserved. 
Ithaca, NY 14853

M. de la Torre Juarez

Jet Propulsion Laboratory, California Institute of Technology

Pasadena, CA 91109

A. R. Vasavada

Jet Propulsion Laboratory, California Institute of Technology

Pasadena, CA 91109

D. Viúdez-Moreiras

Centro de Astrobiología (INTA-CSIC)

Torrejón de Ardoz

28850 Madrid, Spain

C. Zeitlin

Leidos

Houston, TX 77258

Maria-Paz Zorzano Mier

Centro de Astrobiología (INTA-CSIC)

Torrejón de Ardoz

Madrid, Spain

Atmospheric Science Group, Division of Space Technology, Department of Computer Science, Electrical and Space Engineering

Luleå University of Technology

Luleå, Sweden

\section{ABSTRACT}

This article is protected by copyright. All rights reserved. 
Mars Science Laboratory (MSL) Curiosity rover observations of the 2018/Mars Year 34 global/planet-encircling dust storm represent the first in situ measurements of a global dust storm with dedicated meteorological sensors since the Viking Landers. The MSL team planned and executed a science campaign lasting approximately 100 martian sols to study the storm involving an enhanced cadence of environmental monitoring using the rover's meteorological sensors, cameras, and spectrometers. Mast Camera $880 \mathrm{~nm}$ optical depth reached 8.5 and Rover Environmental Monitoring Station measurements indicated a 97\% reduction in incident total UV solar radiation at the surface, $30 \mathrm{~K}$ reduction in diurnal range of air temperature, and an increase in the semidiurnal pressure tide amplitude to $40 \mathrm{~Pa}$. No active dust lifting sites were detected within Gale Crater and global and local atmospheric dynamics were drastically altered during the storm. This work presents an overview of the mission's storm observations and initial results. 


\section{INTRODUCTION}

Global, or planet-encircling, dust storms are an enigmatic feature of the martian climate. For our purposes, the terms "planet-encircling" and "global" are equivalent and we use "global" for this work. Global dust storms typically occur every few Mars years (MY) and persist for weeks to months (we utilize the martian calendar of Clancy et al. [2000]). They radically alter atmospheric circulation [Conrath, 1975; Leovy and Zurek, 1979; Haberle et al., 1982; Zurek, 1982; Wilson, 1997; Newman et al., 2002; Guzewich et al., 2014], change surface albedo patterns [e.g., Szwast et al., 2006; Cantor, 2007; Vincendon et al., 2015], and modify subsequent transport and deposition of water and $\mathrm{CO}_{2}$ at the poles [Benson and James, 2005; Strausberg et al., 2005; Cantor, 2007].

The 2018/MY34 storm began as "arcuate" or frontal-like dust storms in the northern hemisphere (e.g., Wang et al., 2003) that ultimately initiated, merged, or expanded dust-lifting activity in the southern hemisphere [Malin et al., 2018a; 2018b, 2018c]. Precursor storms formed in mid-May $2018, \mathrm{~L}_{\mathrm{s}} \sim 181^{\circ}$ (" $\mathrm{L}_{\mathrm{s}}$ " is areocentric solar longitude and $\mathrm{L}_{\mathrm{s}}=0^{\circ}$ represents northern spring equinox), across Acidalia Planitia (30-60 N, 300-360 E) and Utopia Planitia (30$60^{\circ} \mathrm{N}, 80-140^{\circ} \mathrm{E}$ ) before expanding to cover much of the northern hemisphere and tropics over the next 2 weeks. By early June, $\mathrm{L}_{\mathrm{s}} \sim 188^{\circ}$, substantial dust lifting was occurring independently in the southern hemisphere and those storms along the receding southern seasonal $\mathrm{CO}_{2}$ polar ice cap (e.g., Toigo et al., 2002) merged with the larger storm along and north of the equator [Malin et al., 2018c; 2018d]. By mid-June, $\mathrm{L}_{\mathrm{s}} \sim$ 193이 $^{\circ}$ the storm was termed "global" [Malin et al., 
2018d; 2018e]. Dust lifting continued sporadically for another 2-3 weeks until early July, $\mathrm{L}_{\mathrm{s}} \sim$ $205^{\circ}$, when the storm began a long decay phase and dust started settling out of the atmosphere [Malin et al., 2018f; 2018g]. By mid-September, $\mathrm{L}_{\mathrm{s}} \sim 250^{\circ}$, dust returned to climatologicallytypical levels for the perihelion season, which we use to define when the storm ended [Malin et al., 2018h].

Curiosity's observations of the storm represent the first in situ observations of a martian global dust storm with dedicated meteorological sensors since the Viking landers in 1977 (e.g., Ryan and Henry, 1979) and the first in situ observations with such sensors near the equator. Section 2 introduces Curiosity's instruments that were employed to study the storm and the science campaign that was conducted by the MSL team. We present our findings in Section 3, which serves as an overview to the mission's observations during the dust storm (and will be substantially augmented by upcoming future papers discussing individual results in greater detail), and Section 4 concludes.

\section{METHODOLOGY}

Curiosity carries a suite of instrumentation to study the climate and meteorology of Gale Crater [Grotzinger et al., 2012; Gómez-Elvira et al., 2012]. During the dust storm, relevant observations were frequently obtained by the Rover Environmental Monitoring Station (REMS) [Gómez-Elvira et al., 2012], the Mast Camera (Mastcam) [Bell et al., 2017], the Navigation Cameras (Navcam) [e.g., Moores et al., 2015; Moore et al., 2016], and the Radiation Assessment 
Detector (RAD) [e.g., Rafkin et al., 2014]. Less frequent observations were obtained by the Chemistry and Camera instrument (ChemCam) [McConnochie et al., 2018].

Prior to the MY33 dust storm season, the MSL science team created a "global dust storm campaign" involving an increased cadence of environmental monitoring, to be initiated in the event that a large dust storm occurred on the planet that could reach "global" status. The campaign was updated in early 2018, prior to the MY34 dust storm season, to account for changes in the rover's instrument and operational posture; primarily, the failure of the last working anemometer board of the REMS wind sensor near MSL mission Sol 1500 [Viúdez et al., 2019a, b]. The campaign was initiated on Sol 2075 (7 June 2018, $\mathrm{L}_{\mathrm{s}}=188.7^{\circ}$ ) and stopped on Sol $2169\left(11\right.$ September 2018, $\left.\mathrm{L}_{\mathrm{s}}=248.2^{\circ}\right)$. During the global dust storm campaign, REMS 1-hour extended blocks were scheduled every 3 hours to measure air and ground temperature, relative humidity, ultraviolet radiation, and air pressure (see Newman et al. [2017] for a description of the typical REMS observation cadence, which includes 1-hour duration "extended blocks" scheduled every 6 hours that slide 1 hour earlier each sol). Mastcam measurements of atmospheric opacity ("tau" and "line-of-sight extinction" observations) [Lemmon et al., 2015] were conducted at least once per sol (with 2 exceptions) from Sol 2072 through Sol 2119 and then at a normal rate ( $\sim 3$ times per week) for the remainder of the storm. Values shown below in Figure 1 are retrieved using the Mastcam $880 \mathrm{~nm}$ and $867 \mathrm{~nm}$ filters. Almost daily Navcam observations of line-of-sight atmospheric extinction [Moores et al., 2015; Moore et al., 2016] and dust devil activity ( 2-3/week) were scheduled in addition to an increased frequency of 
ChemCam passive sky observations to monitor dust particle properties and atmospheric gas abundances [McConnochie et al., 2018]. Due to Curiosity's radioisotope thermoelectric generator power source, science operations were not precluded or reduced during the dust storm.

The scientific goals of the campaign included, but were not limited to:

1. How does the meteorological environment within Gale Crater change before and during a global dust storm? What is the signature of global atmospheric dynamics on the local environment via pressure, temperature, and humidity?

2. How does the abundance, vertical distribution, and physical characteristics of atmospheric dust evolve during a storm?

3. Are aeolian processes altered during a storm?

4. Is the local water cycle altered by the storm? If so, in what way?

\section{RESULTS}

\subsection{Column-integrated atmospheric dust opacity}

Atmospheric dust loading is a major driver of the dynamics and circulation of the martian atmosphere and higher dust loading intensifies the circulation [e.g., Zurek and Leovy, 1981; Haberle et al., 1982; Zurek, 1982; Wilson and Hamilton, 1996; Wilson, 1997; Newman et al., 2002]. Therefore, understanding dust opacity during the storm contextualizes all other meteorological observations. Previous in situ measurements of column dust opacity during martian global dust storms came from the Viking landers in 1977/MY12 and the Mars 
Exploration Rovers Spirit and Opportunity in 2007/MY28. Pollack et al. [1979] found the (670 nm) dust opacity to be 2.7-3.2 and 3.7-9 (representing the lower and upper limits of the maximum opacity during the storms) for the two global dust storms observed by Viking Lander 1. During the 2007/MY28 storm the Spirit rover saw $(880 \mathrm{~nm})$ dust opacities reach 4.3 , while Opportunity reached 4.6 and possibly 5 [Lemmon et al., 2015].

As the storm grew between $\mathrm{L}_{\mathrm{s}}=180-190^{\circ}$, dust opacity was modest in Gale Crater with values as low as 0.57 on Sol $2073\left(\mathrm{~L}_{\mathrm{s}}=188.3^{\circ}\right)$ and generally between 0.6 and 0.7 (Figure 1). This is comparable to observed MY33 values and about 0.1 less than in MY32.

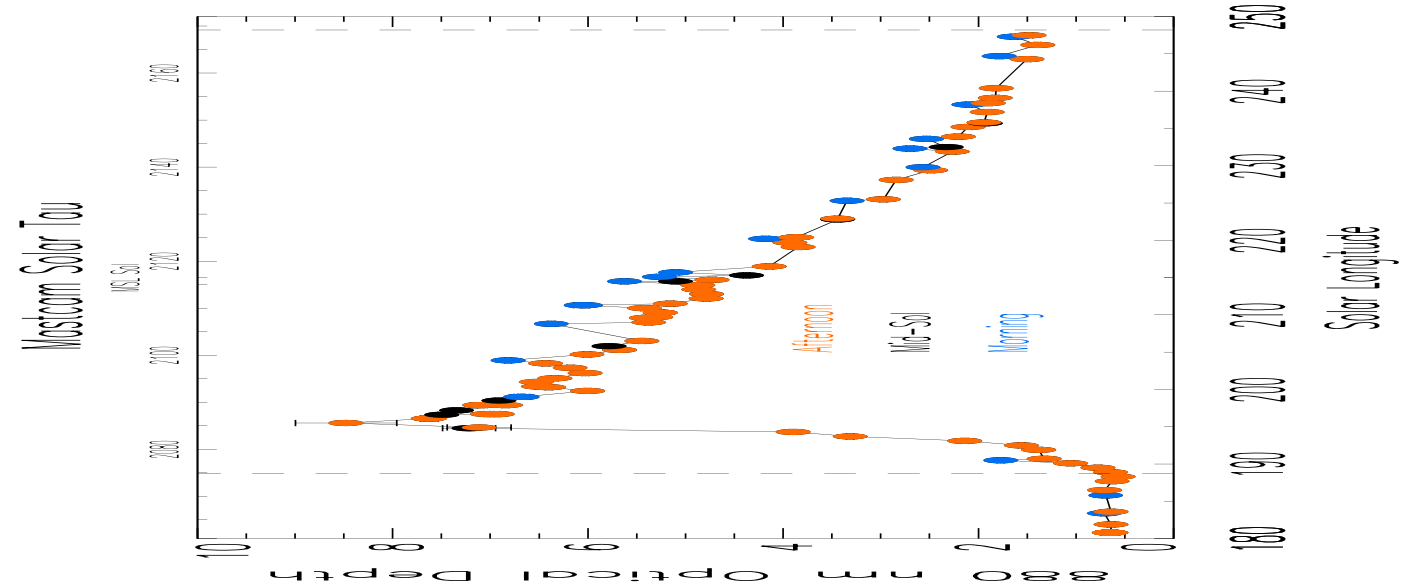

Figure 1: Mastcam $880 \mathrm{~nm}$ atmospheric optical depth during the 2018/MY34 global dust storm plotted against solar longitude and MSL mission sol number. One-sigma error bars are plotted for all points (but only visible for a few points). Points are color-coded based on local true solar time (LTST) of observation with "Morning" defined as 0700-1000 LTST, "Mid-Sol" as 10001400 LTST, and "Afternoon" as after 1400 LTST. Dashed-vertical lines indicate when the MSL global dust storm campaign started and stopped.

This article is protected by copyright. All rights reserved. 
Substantial dust from the storm reached Gale Crater on Sols 2076 and $2077\left(\mathrm{~L}_{\mathrm{s}}=190.1-\right.$ $190.5^{\circ}$ ), when opacity climbed from 0.78 on Sol 2075 to 1.77 on Sol 2077 (Figure 1). The most dramatic increase in dust opacity occurred between Sols 2080 and $2085\left(\mathrm{~L}_{\mathrm{s}}=192.5-195.5^{\circ}\right)$ when opacity climbed from 1.56 to the peak of 8.5 (Figure 1). Orbital observations by the Mars Color Imager (MARCI) indicate this rapid change in dust opacity over Gale was linked to the global evolution of the storm. The initial rise in dust opacity between Sols 2075 and 2080 appears due to the dissipation of a local-scale dust storm southwest of Gale Crater in addition to transport of dust globally from the more intense dust lifting in the opposite hemisphere [Malin et al., 2018d]. Between Sols 2080 and 2085, dust lifting near Hellas Basin, Tyrrhena Terra, and Hesperia Planum expanded and encompassed Gale Crater [Malin et al., 2018d]. Orbital and rover perspectives indicate the majority of dust observed in Gale Crater was advected from elsewhere, i.e., from locations where active dust lifting was observed [Malin et al., 2018d; 2018e].

Although the storm did not peak globally (i.e., major dust lifting did not cease) for several more weeks [Malin et al., 2018g], opacity in Gale Crater began to decline immediately after the dust opacity peak on Sol 2085. Starting on Sol 2107, when sedimentation-only processes appear to begin, through Sol 2171, Curiosity observed an opacity exponential decay timescale of $43 \pm 2$ sols, identical to the 43 sols of the Mars Exploration Rovers during the 
2007/MY28 storm [Lemmon et al., 2015] and close to the 51 sols observed by Viking Lander 1 during the second global storm of 1977/MY12 [Pollack et al., 1979].

Primarily during the first month of the campaign, multiple Mastcam tau observations were often taken on the same sol to search for short-term opacity variation. Variation of $\sim 0.1$ 0.25 (above the 1-sigma level) was seen over 1-3 hours during the mid-sol and afternoon periods.

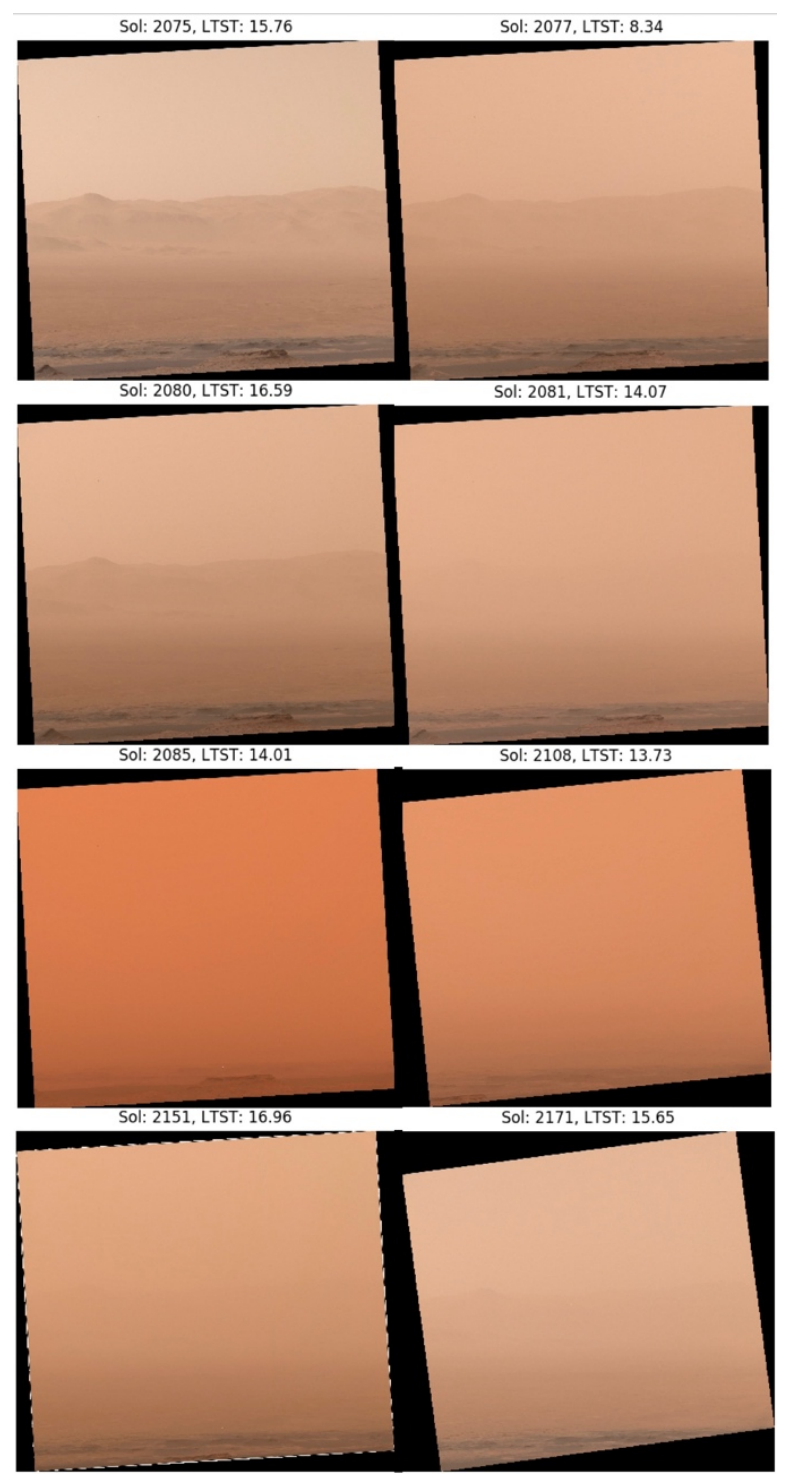


Figure 2: Mastcam "crater rim line-of-sight extinction" images identified by sol number and local true solar time.

\subsection{Line-of-sight atmospheric dust opacity}

In addition to the column-integrated opacity, Curiosity regularly measures the in-crater line-of-sight opacity by imaging the north rim of Gale Crater with Mastcam and Navcam [Moores et al., 2015; Moore et al., 2016]. While the column opacity has occasionally high solto-sol variability [e.g., Smith et al., 2016], the in-crater opacity typically exhibits less transient behavior and often a time lag between column and corresponding in-crater opacity increases [e.g., Guzewich et al., 2017]. As the storm approached Gale, Curiosity was parked near the north edge of the Vera Rubin Ridge. This gave a consistent view for the Mastcam crater rim extinction images shown in the first three rows of Figure 2. By the end of the storm (bottom row of Figure 2), the rover had moved elsewhere, but the viewing angle and range did not appreciably change.

For a sense of scale, the north crater rim that can be clearly seen in the first row of Figure 2 is approximately $30 \mathrm{~km}$ distant from the rover, while the Bagnold dune fields and bluffs are 2-5 $\mathrm{km}$ distant. The high horizontal opacity during the storm broke some of the assumptions used to calculate line-of-sight extinction by Moores et al. [2015] and Moore et al. [2016]. Horizontal visibility is a standard terrestrial weather variable reported to provide information about obscurations in the air and is marked by human observers as the distance to the farthest discernable object. That value fell to approximately $2.7 \mathrm{~km}$ on Sol 2094. Note that this is 9 sols 
after the maximum in column optical depth (Figure 1), suggesting that the bulk of atmospheric dust did advect into Gale Crater from elsewhere (and hence was above the boundary layer within the crater) before settling to lower altitudes with time. By Sols 2151 and 2171 (bottom row in Figure 2), features in the crater rim can be seen again.

\subsection{Meteorological measurements}

Figure 3 provides an overview of meteorological variables measured by REMS and Figure 3a displays the diurnal pressure cycle at three representative times during the growth and onset of the storm: Sol 2053 (before the precursor storms formed in the northern hemisphere); Sol 2074 (as the storm was rapidly growing and encroaching on Gale); and Sol 2096 (after the storm was termed "global" and dust opacity in Gale was very high, $\sim 6$ ). There are two roughly equal contributions to the diurnal pressure cycle at Gale Crater under normal dust conditions. Roughly half the amplitude is provided by the large-scale thermal tides, of which the diurnal mode dominates [Guzewich et al., 2016]. The other half is provided by daily redistribution of atmospheric mass over the complex mesoscale terrain of Gale Crater [Richardson and Newman, 2018]. The changes depicted in Figure $3 \mathrm{a}$ are thus driven by the variation in both amplitudes of the pressure tides and the mesoscale circulation. The diurnal pressure component is most sensitive to dust at the observation location and relatively insensitive to global atmospheric dust loading [Guzewich et al., 2016]. Prior to the storm (asterisks in Figure 3a), the diurnal pressure amplitude (the source of most of the variability seen during a sol) was near $32 \mathrm{~Pa}$, which is seasonally typical. As dust encroached on Gale Crater on Sol 2083 (not shown), the diurnal 
amplitude reached $48 \mathrm{~Pa}$ before returning to climatological values of $30-40 \mathrm{~Pa}$ afterwards. The diurnal amplitude during the storm is likely dominated by the tide rather than the mesoscale "hydrostatic adjustment" circulation (see subsequent paragraphs). The semidiurnal variation, which is almost entirely tidal and highly sensitive to globally-integrated atmospheric dust forcing [e.g., Wilson and Hamilton, 1996; Bridger and Murphy, 1998], rose to planetary record levels and closely tracked the expansion of the storm in the southern hemisphere. Prior to $\mathrm{L}_{\mathrm{s}}=189^{\circ}$, the semidiurnal tide was near climatological values of $\sim 12-14 \mathrm{~Pa}$, before rapidly increasing to $\sim 40 \mathrm{~Pa}$ on Sol $2095 / \mathrm{L}_{\mathrm{s}}=201.5^{\circ}$.
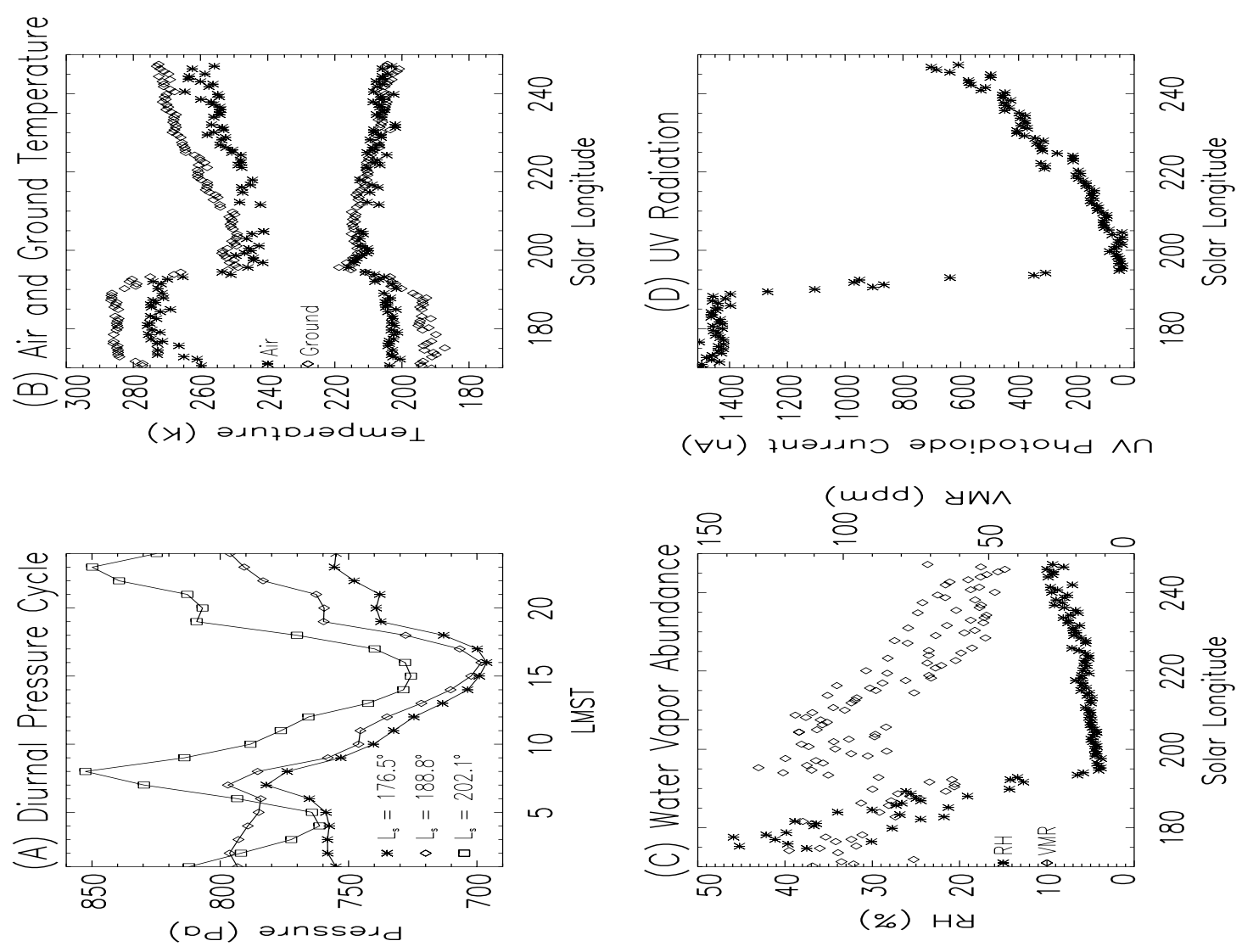

This article is protected by copyright. All rights reserved. 
Figure 3: REMS observations of (A) the diurnal air pressure cycle, (B) the diurnal maximum and minimum air and ground temperatures, (C) the diurnal maximum measured relative humidity (generally achieved between 04:00 and 06:00 LTST) and water vapor abundance obtained at the same time as relative humidity, and (D) the diurnal maximum UV photodiode current.

Diurnal temperature variation decreased drastically in the storm, with typical pre-storm maximum-minimum air and ground temperature ranges of $\sim 70$ and $\sim 90 \mathrm{~K}$, respectively $\left(\mathrm{L}_{\mathrm{s}}<\right.$ $185^{\circ}$ in Figure $3 \mathrm{~b}$ ), declining to $\sim 30$ and $\sim 35 \mathrm{~K}$ during the storm. Prior to the storm, there was also a clear difference between air and ground maximum and minimum temperatures, with the ground temperature typically $5-10 \mathrm{~K}$ cooler at night and $10-15 \mathrm{~K}$ warmer during the daytime (diamonds in Figure 3b). High atmospheric dust opacity warms overnight low temperatures due to increased downwelling infrared radiation [Martínez et al., 2017], resulting in nighttime minimum air and ground temperatures (both occurring near 05:00 local true solar time) 10 and $\sim 20 \mathrm{~K}$ warmer than pre-storm values, respectively. Daytime maximum air and ground temperatures (occurring around 14:00-15:00 and 12:00-13:00 LTST, respectively) were depressed by $\sim 30$ and $\sim 35 \mathrm{~K}$ due to diminished incoming solar radiation. During the storm, the nighttime ground temperatures closely matched the nighttime air temperatures, implying isothermal conditions in the lowest $1.5 \mathrm{~m}$ of the atmosphere (as was anticipated). Daytime ground temperatures also closely followed the air temperature, but remained $\sim 5 \mathrm{~K}$ warmer. As dust opacity declined, the normal differences between daytime air and ground temperatures 
returned, but the nighttime similarity remained past $L_{s}=240^{\circ}$. Daily average air temperature decreased by approximately $10 \mathrm{~K}$ relative to pre-storm values.

The diurnal air temperature range reduction of just over $50 \%$ will have generated a similar fractional reduction in the amplitude of the non-tidal diurnal pressure cycle at the rover. From modeling of the daily pressure cycle under non-storm conditions, it has been shown that about $15 \mathrm{~Pa}$ of the $\sim 32 \mathrm{~Pa}$ observed diurnal pressure amplitude is due to the daily local hydrostatic rebalancing flow [See Figure 5a and Section 4.3 of Richardson and Newman, 2018]. As the relationship between the daily non-tidal pressure amplitude and the daily temperature range at the Curiosity site is linear [see Section 6.4 of Richardson and Newman, 2018], the roughly 50\% drop in the air temperature cycle during the storm will have reduced the daily nontidal pressure amplitude to $\sim 8 \mathrm{~Pa}$. Thus, of the $\sim 48 \mathrm{~Pa}$ total diurnal pressure amplitude observed at the storm peak, the large-scale tide was likely responsible for about $\sim 40 \mathrm{~Pa}$, representing an increase of the diurnal thermotidal amplitude from $\sim 15 \mathrm{~Pa}$ to $\sim 40 \mathrm{~Pa}$ due to the storm.

The REMS measurements of relative humidity are the first performed during a global dust storm (Figure 3c). Orbital observations of previous global storms have hinted that the atmosphere exhibits a lower water content during and after global dust storms as atmospheric temperatures warm [Smith et al., 2002; Fedorova et al., 2018; Smith et al., 2018]. Figure 3c shows that daily maximum relative humidity (asterisks, $\mathrm{RH}$ ) dropped to $\sim 5 \%$ as the storm encompassed Gale Crater $\left(\mathrm{L}_{\mathrm{s}}>195^{\circ}\right)$, largely due to warmer overnight minimum temperatures (Figure 3b). As the storm abated, daily maximum RH values returned to seasonally-typical 
values of $\sim 10 \%$. Figure $3 \mathrm{c}$ also shows water vapor volume mixing ratio (VMR) values obtained at the same time as $\mathrm{RH}$ using concurrent REMS RH, temperature, and atmospheric pressure [Martínez et al., 2017]. The decreasing VMR values between $\mathrm{L}_{\mathrm{s}}=170^{\circ}$ and $190^{\circ}$ (just prior to the storm reaching Gale) also occurred in previous years. Interestingly, neither the sudden increase between $L_{\mathrm{s}}=190^{\circ}$ and $195^{\circ}$ nor the following decreasing trend were observed in previous years. A detailed study of these phenomena will be presented in future work.

Solar flux at the surface was substantially curtailed during the storm. Figure $3 \mathrm{~d}$ shows the daily maximum UV photodiode current, corresponding to the maximum detected UV solar flux, for the REMS UVABC channel before and during the storm. Note that we present UV photodiode current (not UV solar flux) as flux values have not been corrected for dust deposition on the REMS UV sensors [Smith et al., 2016; Vicente-Retortillo et al., 2017] and for inaccuracies in the angular response of the sensors [Vicente-Retortillo et al., 2017]. That correction will be presented in future work. Prior to the storm, daily maximum UVABC photodiode currents were typically $1400-1500 \mathrm{nA}$ before falling to values as low as $40 \mathrm{nA}$ when the highest opacity values were observed in Gale on Sols 2085-2100. Neglecting dust deposition blocking additional solar radiation, this implies a $~ 97 \%$ reduction in incident total UV solar radiation (direct plus diffuse) at the surface during the storm relative to previous years at the same season (i.e., when atmospheric optical depth was approximately 0.7). Note that at UV wavelengths, the single scattering albedo is lower than at visible and near-infrared wavelengths [Wolff et al., 2009; Wolff et al., 2010], resulting in greater absorption. 


\subsection{Vortex and dust devil activity}

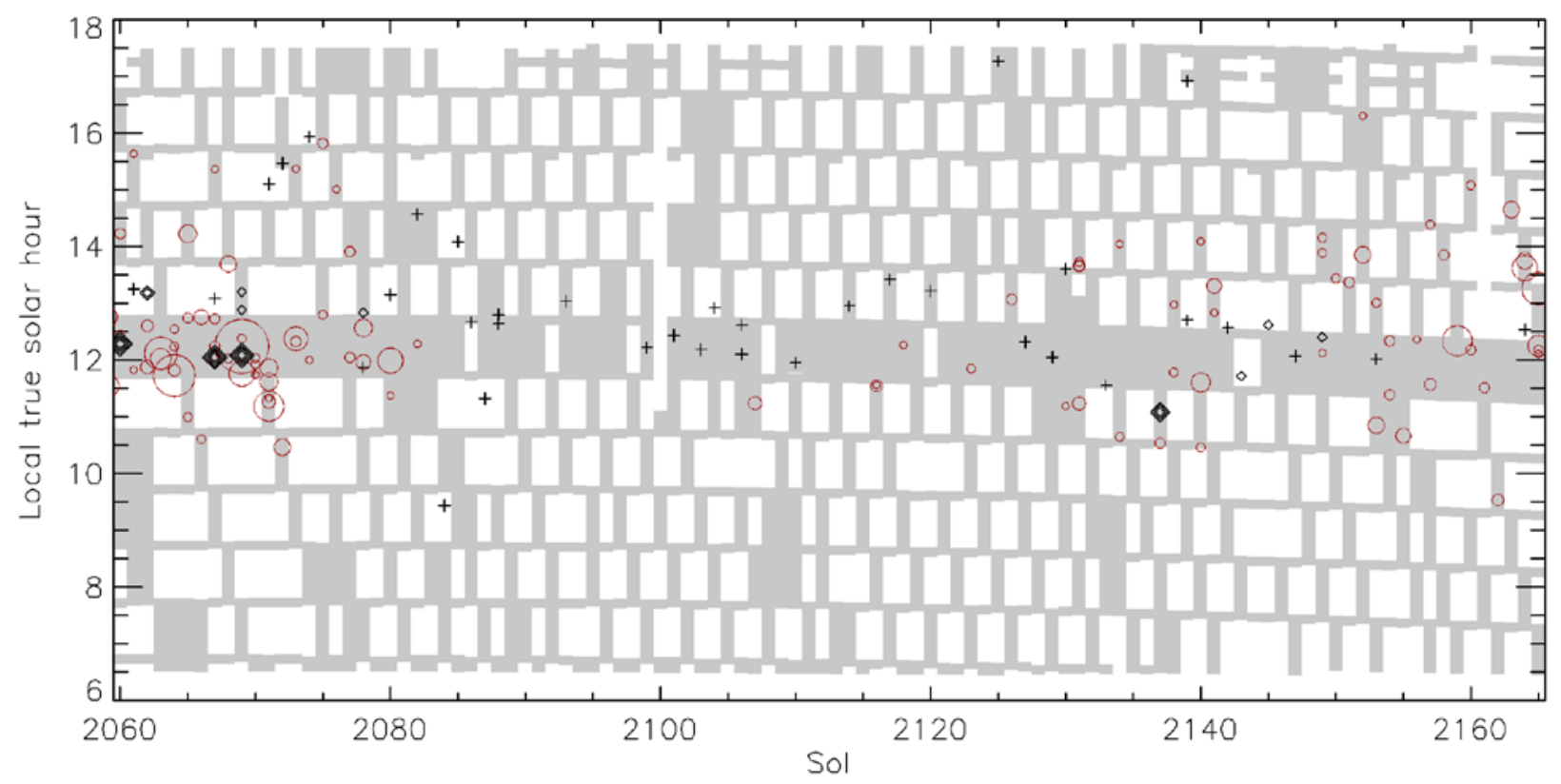

Figure 4: Observed dust devils and REMS pressure vortices before, during, and immediately after the dust storm plotted against local true solar time and sol. Gray shading shows time periods with REMS observations. Red circles indicate a detected REMS pressure vortex, with larger circles indicating a pressure drop > 1.5 Pa. Crosses indicate Navcam "dust devil" observations in which no dust devils were detected, while diamonds show observations with detections (each layer of diamond corresponds to an additional detection within the observation).

Early in the MSL mission, only one candidate dust devil was directly imaged [Moores et al., 2015] despite numerous convective vortices detected with REMS pressure measurements [Kahanpää et al., 2016; Steakley and Murphy, 2016; Ordóñez-Etxeberria et al., 2018]. In the 
past 1.5 Mars years, altered dust devil search sequence pointing and the rover's physical proximity to locations that appear more conducive to dust devils have resulted in regular and numerous dust devil detections within the foothills around Mt. Sharp [Lemmon et al., 2017].

Newman et al. [2002] showed, using the Renno et al. [1998] thermodynamic theory, that increased dust loading is expected to have a negative feedback on dust devil activity due to the reduction in sensible heat flux driven by the near-surface atmospheric temperature gradient. Ignoring changes to the wind conditions, the reduced temperature gradient drove an approximately $80 \%$ reduction in sensible heat flux during the height of the storm near Sol 2085.

Dust devils and convective vortices are seasonally more abundant in southern hemisphere spring and summer and the expected seasonal increase of dust devils was observed prior to the onset of the global dust storm (i.e., before Sol 2070). However, once the dust opacity increased, dust devils/convective vortices ceased entirely within Gale Crater from Sol 2082 through 2106 (Figure 4). One convective pressure vortex was detected on Sol 2107 and then more regular detections resumed after Sol 2116. The first imaged dust devil was not seen until Sol 2137 when opacity decreased to 2.84. Dust devil and pressure vortex frequency was substantially reduced below pre-storm values through mid-September $\left(\mathrm{L}_{\mathrm{s}} \sim 250^{\circ}\right)$.

\subsection{Other results}

We briefly mention additional results that will be expanded upon in future work. A series of Mastcam sky survey observations were conducted to examine dust particles properties and their potential variation during the storm. Smith and Wolff [2014] describe the methodology for 
determining dust particle effective radii. We find that dust effective radius increased from 1.4 $\mu \mathrm{m}$ prior to the storm on Sol 2065 to greater than $4 \mu \mathrm{m}$ on Sol 2097 when optical depth was $>6$. This corroborates work during previous global dust storms that indicates larger particles are lifted by the storm [e.g., Clancy et al., 2010]. Modeling of the surface thermally-derived albedo (from REMS ground temperature measurements, see Figure 3b) shows that the surface albedo increased from 0.2 to 0.28 due to a thin dust coating while thermal inertia was unchanged (see Vasavada et al. [2017] for details on the methodology to produce this result).

"Change detection imaging" with Mastcam has been used previously to assess winddriven motion of surface sediment [Bridges et al., 2017; Baker et al., 2018a,b]. Change detection images were acquired at three sites during the dust storm ("Duluth", "Voyageurs", and "Stoer") to constrain the storm's effect on surface winds around Curiosity. The tail-end of the Duluth imaging campaign revealed substantial sediment motion between Sols 2083 and 2084, just preceding the Sol 2085 opacity peak. Still, this level of motion was not inconsistent with changes during this season of MY33 [Baker et al. 2018b]. Subsequent images obtained at Voyageurs (Sols 2110 - 2114) and Stoer (Sols 2136-2152) indicated moderate levels of aeolian activity, including a small amount of impact ripple migration. Analogous ripple migration has been observed in other instances along the rover's traverse, indicating that the aeolian landscape in Gale Crater may be more controlled by seasonally active background winds than by episodic events [Bridges et al., 2017; Baker et al., 2018a, b]. 


\section{CONCLUSIONS}

For approximately 100 martian sols, the MSL science team conducted an intensive science campaign to study the 2018/MY34 global dust storm involving an increased cadence of environmental monitoring activities with Curiosity's instruments.

Atmospheric optical depth measured by Mastcam solar imaging increased from pre-storm values near 0.6 to a peak of 8.5 over just 12 sols $\left(\mathrm{L}_{\mathrm{s}}=188-196^{\circ}\right)$. Subsequently, opacity began a steady decline at a decay rate of $43 \pm 2$ sols, very similar to decay rates observed by previous landed missions (e.g., Mars Exploration Rovers and Viking Landers). Comparison of column optical depth and in-crater line-of-sight optical depth observed by Mastcam and Navcam indicate that much or all of the dust was sourced from outside of Gale Crater. There were no direct indications of dust lifting within Gale Crater during the storm between Sols 2079 and 2138.

The dust attenuated approximately $97 \%$ of the incident total (direct plus diffuse) UV solar radiation at the surface, leading to a $\sim 40$ and $\sim 55 \mathrm{~K}$ reduction in diurnal range of air and ground temperature, respectively, which resulted in nearly isothermal conditions between the surface and lower atmosphere at night and reduced values of midsol thermal gradient. Such conditions reduced the daytime sensible heat flux, leading to a complete absence of dust devils and convective pressure vortices during the height of the storm.

The REMS pressure sensor observed enhanced atmospheric tides, with the semidiurnal tide reaching amplitudes of $40 \mathrm{~Pa}$, nearly quadrupling climatological values for early southern 
hemisphere spring. The diurnal pressure cycle was also enhanced, with the tidal contribution increasing by a factor of $\sim 3$, while the local mesoscale contribution likely decreased by $\sim 50 \%$.

Surface change detection images did not reveal any significant change to aeolian transport relative to previous years, supporting the overarching idea that the aeolian landscape on Mars is shaped more by gradual processes than by episodic events.

\section{Acknowledgements}

This work was funded by a MSL Participating Scientist grant to S. Guzewich. A portion of the research was carried out at the Jet Propulsion Laboratory, California Institute of Technology, under a contract with the National Aeronautics and Space Administration. MSL data is freely available on the Planetary Data System within six months after receipt on Earth. Data used to make the figures is included in the supplemental material. Mastcam and Navcam images are freely available at https://mars.nasa.gov/msl/multimedia/raw/. We thank two anonymous reviewers for their helpful comments which have improved this manuscript.

\section{REFERENCES}

Baker, M. M., Newman, C. E., Lapotre, M. G. A., Sullivan, R., Bridges, N. T., \& Lewis, K. W. (2018a). Coarse sediment transport in the modern Martian environment. Journal of Geophysical Research: Planets, 123, 1380-1394. https://doi.org/10.1002/2017JE005513

Baker, M. M., Lapotre, M. G. A., Minitti, M. E., Newman, C. E., Sullivan, R., Weitz, C. M., et al. (2018b). The Bagnold Dunes in southern summer: Active sediment transport on Mars observed by the Curiosity rover. Geophysical Research Letters, 45, 88538863. https://doi.org/10.1029/2018GL079040 
Bell, J. F., III, A. Godber, S. McNair, M. A. Caplinger, J. N. Maki, M. T. Lemmon, J. Van Beek, M. C. Malin, D. Wellington, K. M. Kinch, M. B. Madsen, C. Hardgrove, M. A. Ravine, E. Jensen, D. Harker, R. B. Anderson, K. E. Herkenhoff, R. V. Morris, E. Cisneros, and R. G. Deen (2017), The Mars Science Laboratory Curiosity rover Mastcam instruments: Preflight and in-flight calibration, validation, and data archiving, Earth and Space Science, 4, 396-452, doi:10.1002/2016EA000219.

Benson, J.L. and P.B. James (2005), Yearly comparisons of the martian polar caps: 1999-2003 Mars Orbiter Camera observations, Icarus, 174 (2), 513-523.

Bridger, A.F.C., and J.R. Murphy (1998), Mars' surface pressure tides and their behavior during global dust storms, J. Geophys. Res.-Planets, 103(3334), 85887-8601.

Bridges, N. T., R. Sullivan, C.E. Newman, S. Navarro, J. van Beek, R.C. Ewing, F. Ayoub, S. Silvestro, O. Gasnault, S. Le Mouélic, M.G.A. Lapotre, and W. Rapin (2017), Martian aeolian activity at the Bagnold Dunes, Gale Crater: The view from the surface and orbit, J. Geophys. Res. Planets, 122, 2077-2110, doi: 10.1002/2017JE005263.

Cantor, B.A. (2007), MOC observations of the 2001 Mars planet-encircling dust storm, Icarus, 186(1), 60-96.

Clancy, R. T., B. J. Sandor, M. J. Wolff, P. R. Christensen, M. D. Smith, J. C. Pearl, B. J. Conrath, and R. J. Wilson (2000), An intercomparison of ground-based millimeter, MGS TES, and Viking atmospheric temperature measurements: Seasonal and interannual variability of temperatures and dust loading in the global Mars atmosphere, J. Geophys. Res., 105(E4), 9553-9571, doi: 10.1029/1999JE001089.

Clancy, R.T., M.J. Wolff, B.A. Whitney, B.A. Cantor, M.D. Smith, and T.H. McConnochie (2010), Extension of atmospheric dust loading to high altitudes during the 2001 Mars dust storm: MGS TES limb observations, Icarus, 207(1), 98-109, https://doi.org/10.1016/j.icarus.2009.10.011.

Conrath, B.J. (1975), Thermal structure of the Martian atmosphere during the dissipation of the dust storm of 1971, Icarus, 24, 36-46.

Fedorova, A., J.-L. Bertaux, D. Betsis, F. Montmessin, O. Korablev, L. Maltagliati, and J. Clarke (2018), Water vapor in the middle atmosphere of Mars during the 2007 global dust storm, Icarus, 300, 440-457, https://doi.org/10.1016/j.icarus.2017.09.025. 
Gómez-Elvira, J., C. Armiens, L. Castañer, M. Domínguez, M. Genzer, F. Gómez, R. Haberle, A.-M. Harri, V. Jiménez, H. Kahanpää, L. Kowalski, A. Lepinette, J. Martín, J. MartínezFrías, I. McEwan, L. Mora, J. Moreno, S. Navarro, M. A. de Pablo, V. Peinado, A. Peña, J. Polkko, M. Ramos, N. O. Renno, J. Ricart, M. Richardson, J. Rodríguez-Manfredi, J. Romeral, E. Sebastián, J. Serrano, M. de la Torre Juárez, J. Torres, F. Torrero, R. Urquí, L. Vázquez, T. Velasco, J. Verdasca, M.-P. Zorzano, J. Martín-Torres (2012), REMS: The Environmental Sensor Suite for the Mars Science Laboratory, Space Science Reviews, 170, 583-640.

Grotzinger, J.P., J. Crisp, A.R. Vasavada, R.C. Anderson, C.J. Baker, R. Barry, D.F. Blake, P. Conrad, K.S. Edgett, B. Ferdowski, R. Gellert, J.B. Gilbert, M. Golombek, J. GómezElvira, D.M. Hassler, L. Jandura, M. Litvak, P. Mahaffy, J. Maki, M. Meyer, M.C. Malin, I. Mitrofanov, J.J. Simmonds, D. Vaniman, R.V. Welch, and R.C. Wiens (2012), Mars Science Laboratory Mission and Science Investigation, Space Science Reviews, 170(1-4), $5-56$.

Guzewich, S. D., R. J. Wilson, T. H. McConnochie, A. D. Toigo, D. J. Banfield, and M. D. Smith (2014), Thermal tides during the 2001 Martian global-scale dust storm, $J$. Geophys. Res. Planets, 119, 506-519, doi: 10.1002/2013JE004502.

Guzewich, S.D., C.E. Newman, M. de la Torre Juarez, R.J. Wilson, M. Lemmon, M.D. Smith, H. Kahanpää, A.-M. Harri and the REMS Science Team and MSL Science Team (2016), Atmospheric tides in Gale Crater, Mars, Icarus, 268, 37-49, doi:10.1016/j.icarus.2015.12.028.

Guzewich, S. D., C.E. Newman, M.D. Smith, J.E. Moores, C.L. Smith, C. Moore, M.I. Richardson, D. Kass, A. Kleinböhl, M. Mischna, F.J. Martín-Torre, M.P. Zorzano-Mier, Battalio, M. (2017). The vertical dust profile over Gale Crater, Mars. Journal of Geophysical Research: Planets, 122, 2779-2792. https://doi.org/10.1002/2017JE005420

Haberle, R.M., C.B. Leovy, J.B. Pollack (1982), Some effects of global dust storms on the atmospheric circulation on Mars, Icarus, 50, 322-367.

Kahanpää, H., C.E. Newman, J.E. Moores, M.-P. Zorzano, J. Martín-Torres, S. Navarro, A. Lepinette, B. Cantor, M.T. Lemmon, P. Valentín-Serrano, A. Ullán, and W. Schmidt (2016), Convective vortices and dust devils at the MSL landing site: Annual variability, J. Geophys. Res. Planets, 121, 1514-1549, doi: 10.1002/2016JE005027.

This article is protected by copyright. All rights reserved. 
Lemmon, M.T., M.J. Wolff, J.F. Bell III, M.D. Smith, B.A. Cantor, and P.H. Smith (2015), Dust aerosol, clouds, and the atmospheric optical depth record over 5 Mars years of the Mars Exploration Rover mission, Icarus, 251, 96-111.

Lemmon, M.T. and Newman, C.E., N. Renno, E. Mason, M. Battalio, M.I. Richardson, and H. Kahanpää (2017), Dust Devil Activity at the Curiosity Mars Rover Field Site, \#2952, presented at the $48^{\text {th }}$ Lunar and Planetary Science Conference, Houston, TX, 2017.

Leovy, C.B., and R.W. Zurek (1979), Thermal tides and Martian dust storms: Direct evidence for coupling, J. Geophys. Res., 84, 2956-2968.

Mal in, M. C., B. A. Cant or, A. W. Br it t on (2018a), MRO MARCI Weat her Report for the week of 21 Ma y 2018-27 May 2018, Mal in Space Science Systems Ca pt ioned Ima ge Rel ea se, MSSS-

532, ht t p://www.mss s.com/mss s_ima ges/2018/05/30/.

Mal in, M. C., B. A. Cant or, A. W. Br it t on (2018b), MRO MARCI Wea ther Report for the week of 28 Ma y 2018-3 June 2018, Mal in Spa ce Science Syst ems Ca pt ioned Ima ge Rel ea se, MSSS-

533, ht t p://www.mss s.com/mss s_ima ges/2018/06/06/.

Mal in, M. C., B. A. Cant or, A. W. Br it t on (2018c), MRO MARCI We a ther Report for the week of 4 June 2018-10 June 2018, Mal in Space Science Syst ems Ca pt ioned Ima ge Rel ea se, MSSS-

534, ht t p://www.ms s s.com/mss s_ima ges/2018/06/13/.

Mal in, M. C., B. A. Cant or, A. W. Br it t on (2018d), MRO MARCI Weat her Report for the week of 11 June 2018 - 17 June 2018, Ma 1 in Space Science Systems Capt ioned Ima ge Rel ea se, MSSS-

535, ht t p://www.mss s.com/mss s_ima ges/2018/06/20/.

Mal in, M. C., B. A. Cant or , A. W. Br it t on (2018e), MRO MARCI Wea ther Report for the week of 18 June 2018 - 24 June 2018, Ma 1 in Space Science Syst ems Capt ioned Ima ge Rel ea se, MSSS-

536, ht t p://www.mss s.com/mss s_ima ges/2018/06/27/.

Mal in, M. C., B. A. Cant or , A. W. Br it t on (2018f), MRO MARCI Weat her Report for the week of 2 Jul y 2018-8 Jul y 2018, Mal in Space Science Syst ems Ca pt ioned Ima ge Rel ea se, MSSS-

538, ht t p://www.mss s.com/mss s_ima ges/2018/07/11/.

This article is protected by copyright. All rights reserved. 
Mal in, M. C., B. A. Cant or, A. W. Br it t on (2018g), MRO MARCI Weat her Report for the week of 16 Jul y $2018-22$ Jul y 2018, Mal in Space Science Syst ems Ca pt ioned Ima ge Rel ea se, MSSS-

540, ht t p://www.mss s.com/mss s_ima ges/2018/07/25/.

Ma 1 in, M.C., B.A. Cant or, A.W. Br it t on (2018h), MCO MARCI Weat her Report for the week of 3 Sept ember 2018-9 Sept ember 2018, Mal in Space Science Syst ems Ca pt io ned Ima ge Rel ea se, MSSS-547,

ht t p://www.ms s s.com/ms s s_ima ges/2018/09/12/.

Martínez, G.M., C.E. Newman, A. De Vicente-Retortillo, E. Fischer, N.O. Renno, M.I. Richardson, A.G. Fairen, M. Genzer, S.D. Guzewich, R.M. Haberle, A.-M. Harri, O. Kemppinen, M.T. Lemmon, M.D. Smith, M. de la Torre-Juarez, A.R. Vasavada (2017), The Modern Near-Surface Martian Climate: A Review of In-situ Meteorological Data from Viking to Curiosity, Space Science Reviews, doi:10.1007/s 11214-017-0360-x

McConnochie, T.H., M.D. Smith, M.J. Wolff, S. Bender, M. Lemmon, R.C. Wiens, S. Maurice, O. Gasnault, J. Lasue, P.-Y. Meslin, A.-M. Harri, M. Genzer, O. Kemppinen, G. M. Martínez, L. DeFlores, D. Blaney, J.R. Johnson, and J.F. Bell III (2018), Retrieval of water vapor column abundance and aerosol properties from ChemCam passive sky spectroscopy, Icarus, 307, 294-326.

Moore, C.A., J.E. Moores, M.T. Lemmon, S.C.R. Rafkin, R. Francis, J. Pla-García, R.M. Haberle, M.-P. Zorzano, F.J. Martín-Torres, J.R. Burton (2016), A full martian year of line-of-sight extinction within Gale Crater, Mars as acquired by the MSL Navcam through sol 900, Icarus, 264, 102-108, doi:10.1016/j.icarus.2015.09.001.

Moores, J.E., et al. (2015), Observational evidence of a suppressed planetary boundary layer in northern Gale Crater, Mars as seen by the Navcam instrument onboard the Mars Science Laboratory rover, Icarus, 249, 129-142, doi:10.1016/j.icarus.2014.09.020.

Newman, C. E., S. R. Lewis, P. L. Read, and F. Forget (2002), Modeling the Martian dust cycle, 1. Representations of dust transport processes, Journal of Geophysical Research: Planets 107, no. E12, 6-1.

Newman, C.E., J. Gómez-Elvira, M.I. Richardson, M. Marín, S. Navarro, J. Torres, M. Battalio, S.D. Guzewich, R. Sullivan, M. de la Torre, and A. Vasavada (2017), Winds measured by the Rover Environmental Monitoring System (REMS) during the Mars Science Laboratory (MSL) rover's Bagnold Dunes campaign and comparison with numerical modeling using MarsWRF, Icarus, 291, 203-231, doi:10.1016/j.icarus.2016.12.016. 
Ordóñez-Etxeberria, I., R. Hueso, and A. Sánchez-Lavega (2018), A systematic search of sudden pressure drops on Gale crater during two Martian years derived from MSL/REMS data, Icarus, 299, 308-330, https://doi.org/10.1016/j.icarus.2017.07.032.

Prats, B., D. Archer, M. Trainer, C. Malespin, S. Teinturier, S.D. Guzewich, P. Mahaffy, M.T. Lemmon, G. Martínez, and K. Gonter (2018), 2018 Mars Global Dust Storm - Effects of Airborne Dust and Particle Deposition on Mars Science Laboratory SAM (Sample Analysis at Mars) Instrument Inlet Cover Actuator Temperatures, presented at the Fall American Geophysical Union Meeting, Washington, DC, December, 2018.

Pollack, J. B., D. S. Colburn, F. M. Flasar, R. Kahn, C. E. Carlston, and D. G. Pidek (1979), Properties and effects of dust particles suspended in the Martian atmosphere, J. Geophys. Res., 84, 2929-2945. doi: 10.1029/JB084iB06p02929.

Rafkin, S. C. R., C. Zeitlin, B. Ehresmann, D. Hassler, J. Guo, J. Köhler, R.WimmerSchweingruber, J. Gómez-Elvira, A.-M. Harri, H. Kahanpää, D.E. Brinza, G. Weigle, S. Böttcher, E. Böhm, S. Burmeister, C. Martin, G. Reitz, F.A. Cucinotta, M.-H. Kim, D. Grinspoon, M.A. Bullock, A. Posner, and the MSL Science Team (2014), Diurnal variations of energetic particle radiation at the surface of Mars as observed by the Mars Science Laboratory Radiation Assessment Detector, J. Geophys. Res. Planets, 119, 13451358, doi: 10.1002/2013JE004525.

Rennó, N.O., M.L. Burkett, and M.P. Larkin (1998), A simple thermodynamic model for dust devils, J. Atmos. Sci., 55, 3244-3252.

Richardson, M.I., and C.E. Newman (2018), On the relationship between surface pressure, terrain elevation, and air temperature. Part I: The large diurnal surface pressure range at Gale Crater, Mars and its origin due to lateral hydrostatic adjustment, Plan. Space Sci., doi.org/10.1016/j.pss.2018.07.003

Ryan, J.A., and R.M. Henry (1979), Mars atmospheric phenomena during major dust storms, as measured at surface, J. Geophys. Res., 84, 2821.

Smit h, M.D., B.J. Conr at h, J.C. Pear l, and P.R. Chr ist en sen (2002), Ther ma 1 emission spectrometer observations of the Martian planetencir cl ing dust st or m 2001 A, Icarus, 157(1), 96797, 259-263.

Smit h, M.D. (2009), THEMIS observation s of Mars a er osol opt ical dept h from 2002-2008, Ic a r us, 202(2), 444-452, ht t ps://d oi.or g/10.1016/j.ic ar us.2009.03.027.

This article is protected by copyright. All rights reserved. 
Smit h, M.D., and M.J. Wol ff (2014), Dust Aer osol Particle Size and Sha pe Using MER Navcam and Pancam Sky Imaging, present ed at the $5^{\text {th }}$

Int er national Workshop on the Mars At mosphere: Modelling and Observat ions, Oxfor d, UK, Januar y, 2014.

Smith, M.D., M.-P. Zorzano, M. Lemmon, J. Martín-Torres, and T. Mendaza de Cal (2016), Aerosol optical depth as observed by the Mars Science Laboratory REMS UV photodiodes, Icarus, 280, 234-248, doi:10.1016/j.icarus.2016.07.012.

Smith, M.D., F. Daerden, L. Neary, and A. Khayat (2018), The climatology of carbon monoxide and water vapor on Mars as observed by CRISM and modeled by the GEM-Mars general circulation model, Icarus, 301, 11-131, https://doi.org/10.1016/j.icarus.2017.09.027.

Steakley, K., and J. Murphy (2016), A year of convective vortex activity at Gale Crater, Icarus, 278, 180-193, doi:10.1016/j.icarus.2016.06.010.

Strausberg, M. J., H. Wang, M. I. Richardson, S. P. Ewald, and A. D. Toigo (2005), Observations of the initiation and evolution of the 2001 Mars global dust storm, J. Geophys. Res., 110, E02006, doi:10.1029/2004JE002361.

Szwast, M. A., M. I. Richardson, and A. R. Vasavada (2006), Surface dust redistribution on Mars as observed by the Mars Global Surveyor and Viking orbiters, J. Geophys. Res., 111, E11008, doi:10.1029/2005JE002485.

Toigo, A. D., M. I. Richardson, R. J. Wilson, H. Wang, and A. P. Ingersoll, A first look at dust lifting and dust storms near the south pole of Mars with a mesoscale model, J. Geophys. Res., 107(E7), doi:10.1029/2001JE001592, 2002.

Vasavada, A.R., S. Piqueux, K.W. Lewis, M.T. Lemmon, and M.D. Smith (2017), Thermophysical properties along Curosity's traverse in Gale crater, Mars, derived from the REMS ground temperature sensor, Icarus, 284, 372-386, https://doi.org/10.1016.j.icarus.2016.11.035.

Vicente-Retortillo, Á., G. M. Martínez, N. O. Renno, M. T. Lemmon, and M. de la Torre-Juárez (2017), Determination of dust aerosol particle size at Gale Crater using REMS UVS and Mastcam measurements, Geophys. Res. Lett., 44, 3502-3508, doi: 10.1002/2017GL072589.

Vincendon, M., J. Audouard, F. Altieri, and A. Ody (2015), Mars Express measurements of surface albedo changes over 2004-2010, Icarus, 251, 145-163, https://doi.org/10.1016/j.icarus.2014.10.029.

This article is protected by copyright. All rights reserved. 
Viúdez-Moreiras, D., Gómez-Elvira, J., Newman, C.E, Navarro, S., Marin, M., Torres, J., de la Torre, M. \& the MSL team. (2019a), Gale Surface Wind Characterization based on the Mars Science Laboratory REMS Dataset. Part I: Wind Retrieval and Gale's Wind Speeds and Directions, Icarus, 319.

Viúdez-Moreiras, D., Gómez-Elvira, J., Newman, C.E, Navarro, S., Marin, M., Torres, J., de la Torre, M. \& the MSL team (2019b), Gale Surface Wind Characterization based on the Mars Science Laboratory REMS Dataset. Part II: Wind Probability Distributions, Icarus, 319, 645-656.

Wang, H., M. I. Richardson, R. J. Wilson, A. P. Ingersoll, A. D. Toigo, and R. W. Zurek (2003), Cyclones, tides, and the origin of a cross-equatorial dust storm on Mars, Geophys. Res. Lett., 30, 1488, doi:10.1029/2002GL016828, 9.

Wilson, R. J. (1997), A general circulation model simulation of the Martian polar warming, Geophysical Research Letters, 24, no. 2, 123-126.

Wilson, R. J., and K. Hamilton (1996), Comprehensive model simulation of thermal tides in the Martian atmosphere, J. Atmos. Sci., 53, 1290-1326, doi:10.1175/15200469(1996)053<1290:CMSOTT>2.0.CO;2.

Wolff, M.J., M.D. Smith, R.T. Clancy, R. Arvidson, M. Kahre, F. Seelos IV, S. Murchie, and H. Savijärvi (2009), Wavelength dependence of dust aerosol single-scattering albedo as observed by the Compact Reconnaissance Imaging Spectrometer. J. Geophys. Res., 114, E00D04, doi: 10.1029/2009JE003350.

Wolff, M.J., R.T. Clancy, J.D. Goguen, M.C. Malin, and B.A. Cantor (2010), Ultraviolet dust aerosol properties as observed by MARCI, Icarus, 208, 143-155, doi: 10.1016/j.icarus.2010.01.010.

Zurek, R., and C. B. Leovy (1981), Thermal tides in the dusty Martian atmosphere: A verification of theory, Science, 213, 437-439, doi:10.1126/science.213.4506.437.

Zurek, R.W. (1982), Martian great dust storms: An update, Icarus, 50, 2-3, 288-310, https://doi.org/10.1016/0019-1035(82)90127-0.

This article is protected by copyright. All rights reserved. 


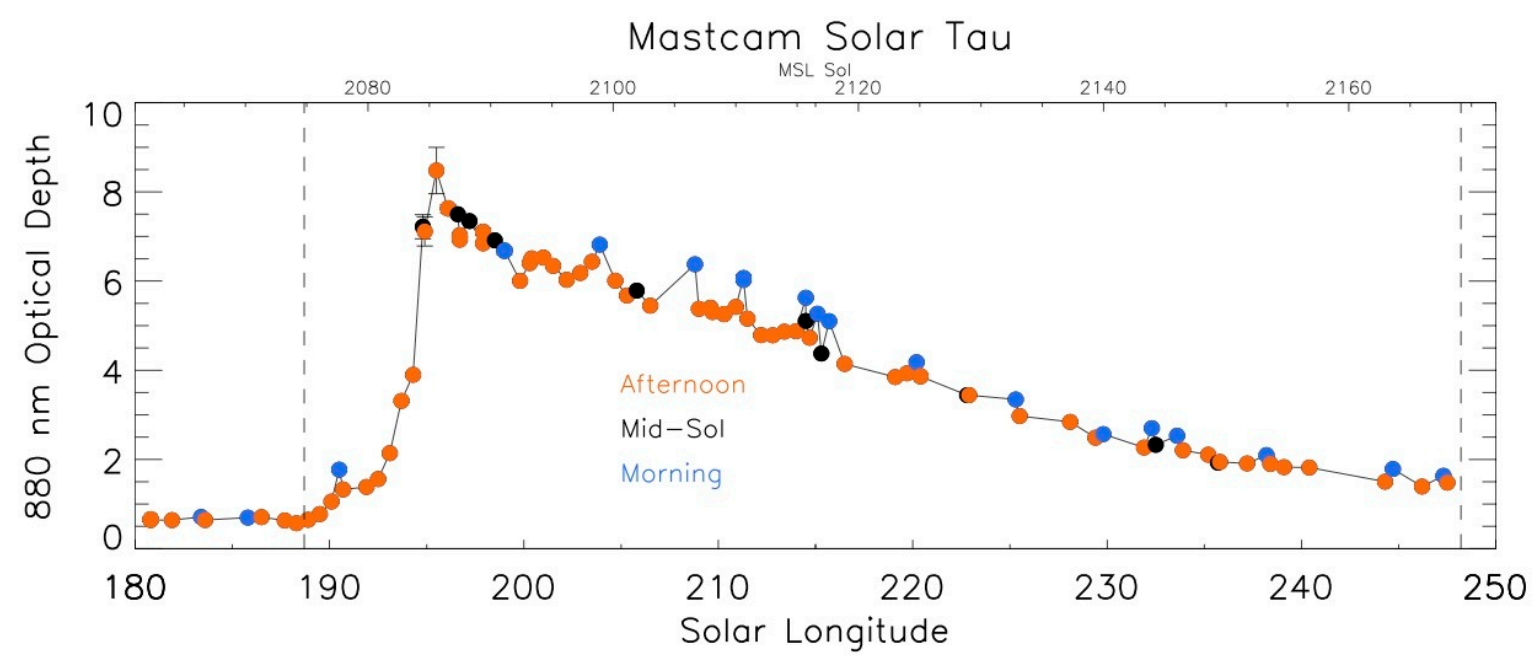

2018GL080839-f01-z-.jpg

This article is protected by copyright. All rights reserved. 
Sol: 2080, LTST: 16.59

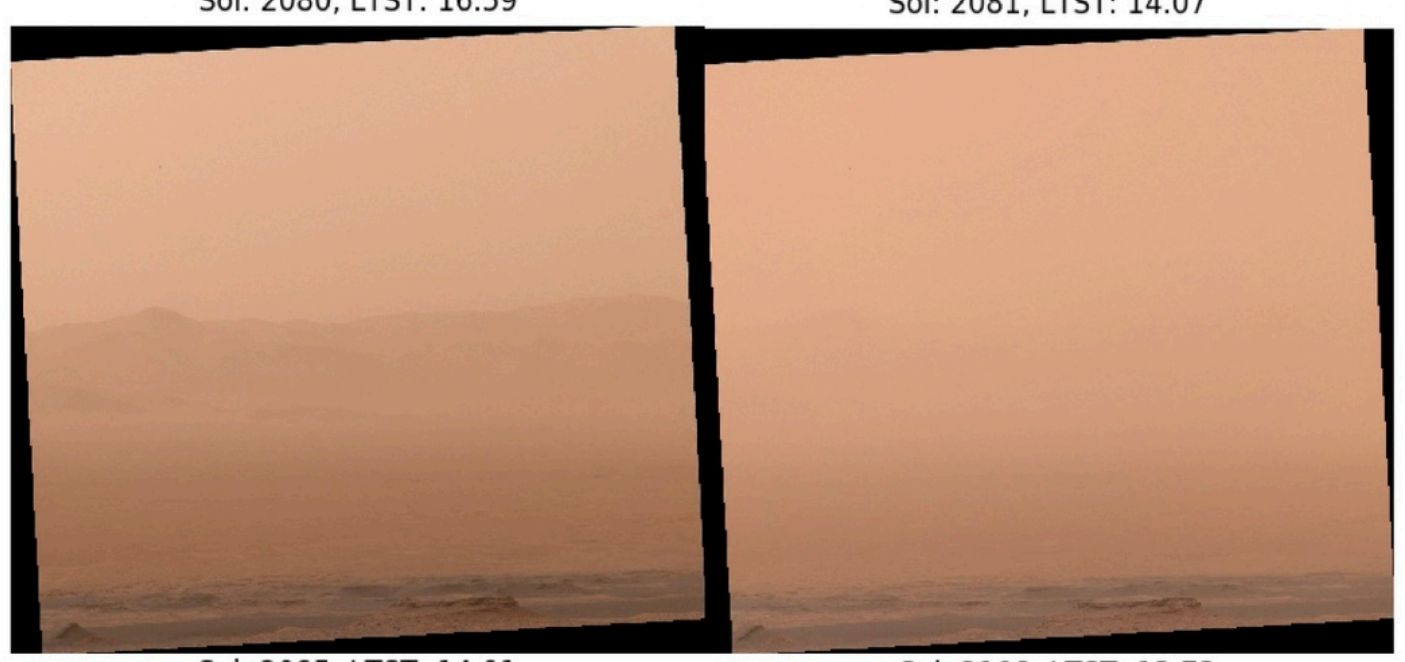

Sol: 2085, LTST: 14.01

Sol: 2108, LTST: 13.73

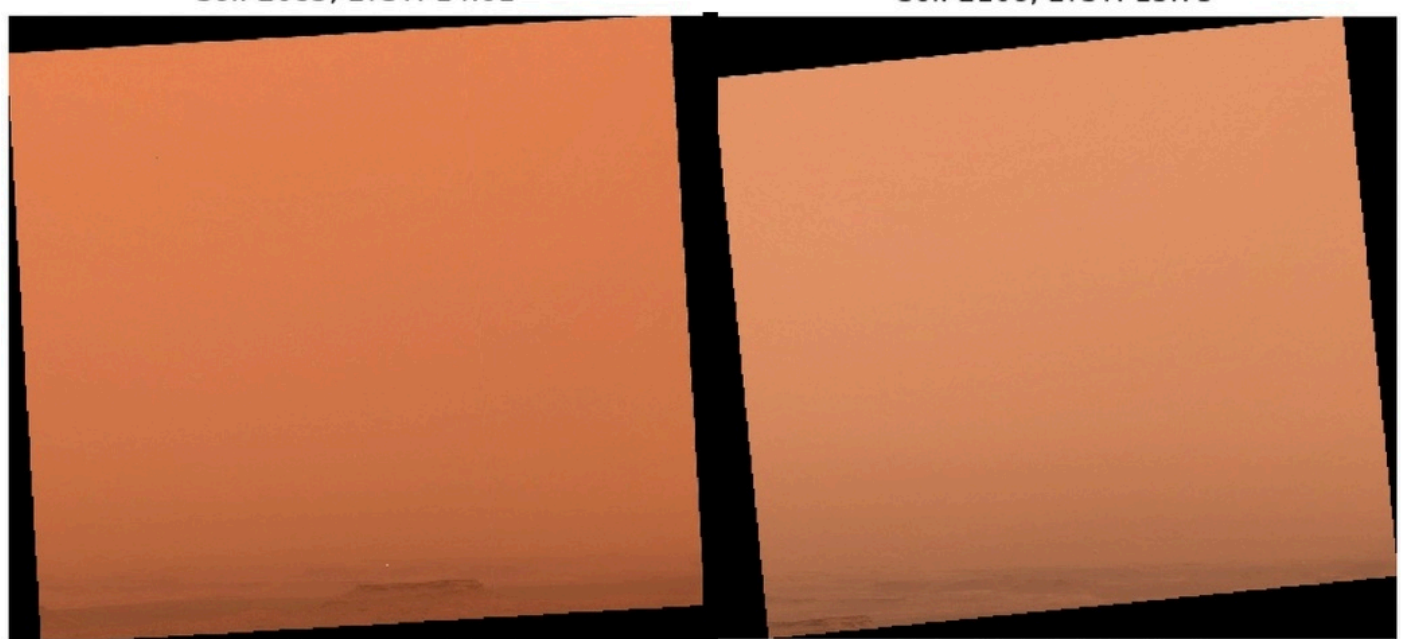

Sol: 2151, LTST: 16.96

Sol: 2171, LTST: 15.65

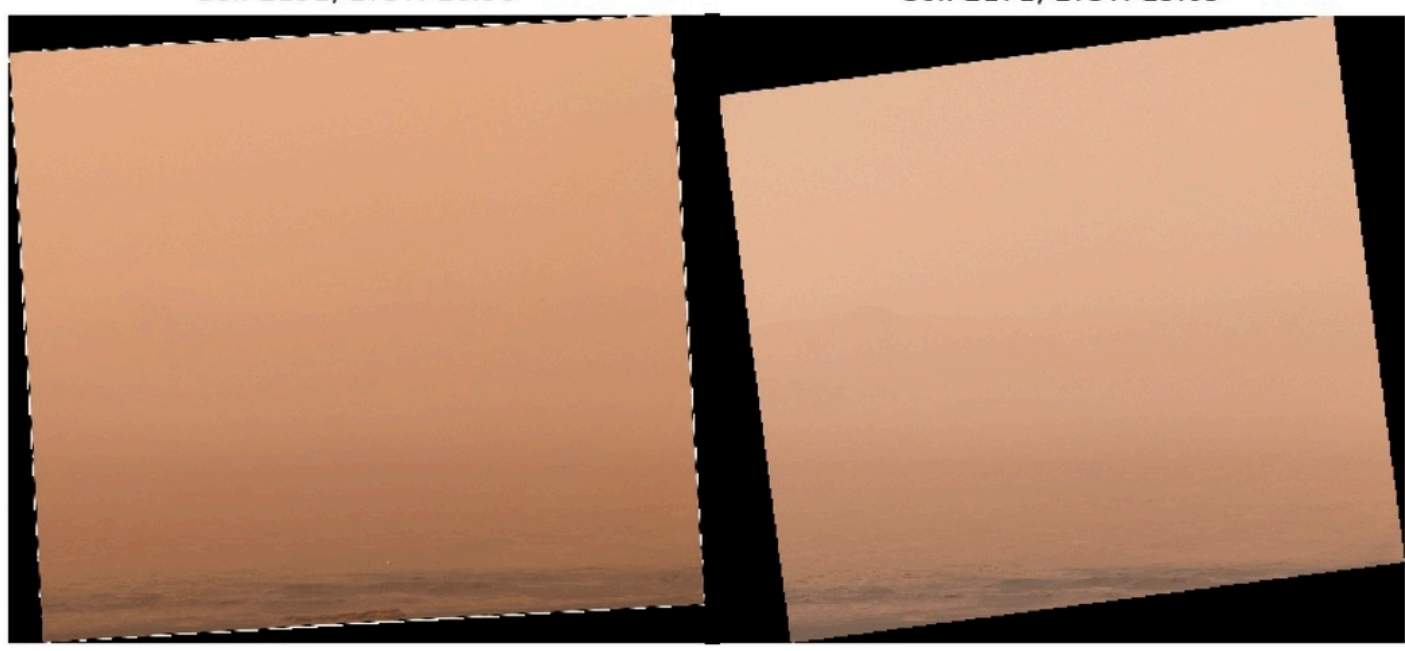

2018GL080839-f02-z-.jpg

This article is protected by copyright. All rights reserved. 

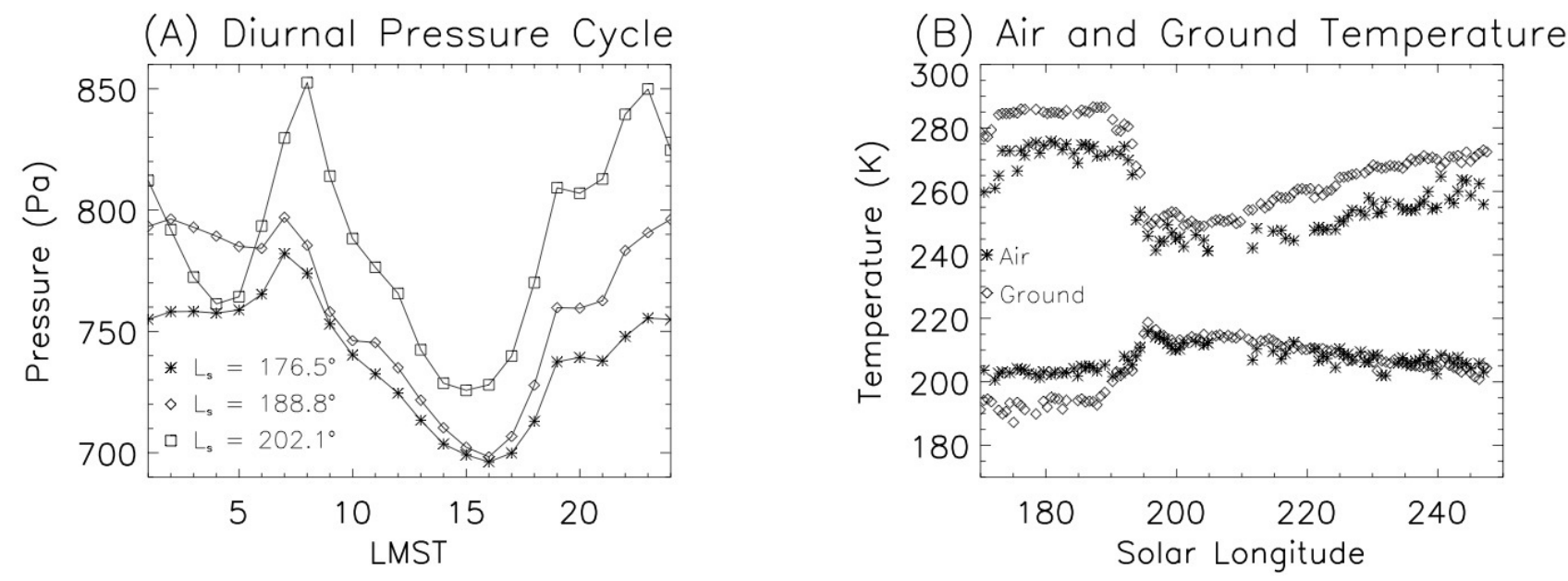

(C) Water Vapor Abundance

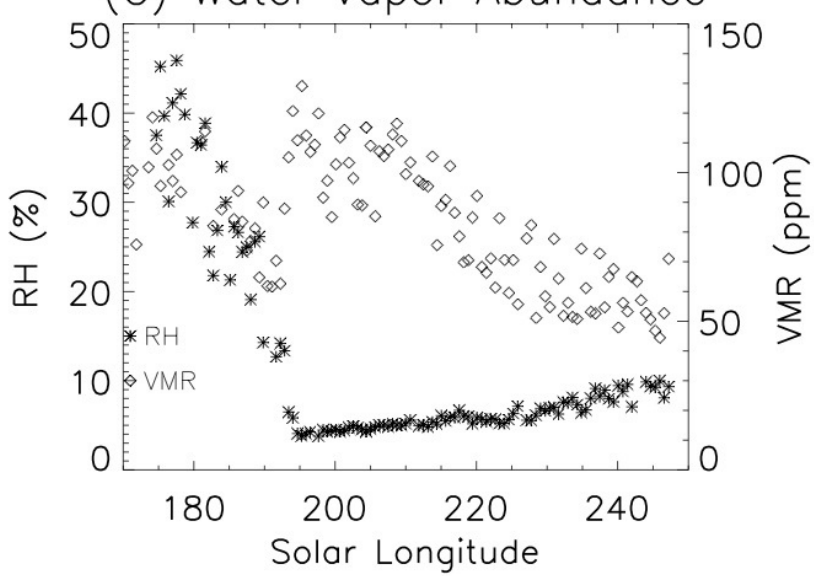

(D) UV Radiation

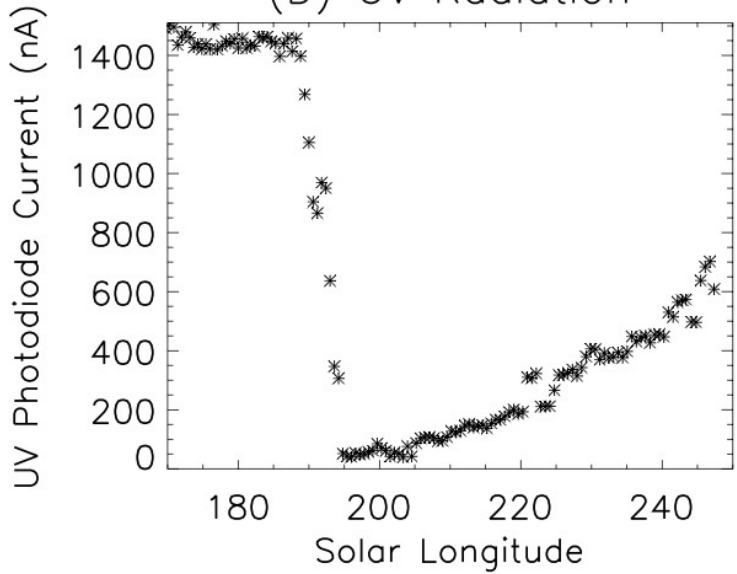

2018GL080839-f03-z-.jpg

This article is protected by copyright. All rights reserved. 
